GANIT J. Bangladesh Math. Soc. (ISSN 1606-3694) 34 (2014) 21-26

\title{
JORDAN DERIVATIONS ON COMPLETELY SEMIPRIME GAMMA-RINGS
}

\author{
Md. Mizanor Rahman ${ }^{1}$ and Akhil Chandra Paul ${ }^{2}$ \\ ${ }^{1}$ Department of Mathematics, Jagannath University, Dhaka-1100, Bangladesh \\ ${ }^{2}$ Department of Mathematics, University of Rajshahi, Rajshahi-6205, Bangladesh \\ ${ }^{1}$ E-mail: mizanorrahman@gmail.com; ${ }^{2}$ E-mail: acpaulrubd_math@yahoo.com
}

Received 29.08.2013 Accepted 10.05.2014

\begin{abstract}
In this paper we prove that under a suitable condition every Jordan derivation on a 2-torsion free completely semiprime $\Gamma$-ring is a derivation.
\end{abstract}

Keywords: Derivation, Jordan derivation, $\Gamma$-ring, completely semiprime $\Gamma$-ring

\section{Introduction}

The concepts of derivation and Jordan derivation of a $\Gamma$-ring have been introduced by $M$. Sapanci and A. Nakajima in [8]. For the classical ring theories, Herstien [6], proved a well known result that every Jordan derivation in a 2-torsion free prime ring is a derivation. Bresar[2] proved this result in semiprime rings. In [8], Sapanci and Nakajima proved the same result in completely prime $\Gamma$-rings. C. Haetinger [4] worked on higher derivations on prime rings and extended this result to Lie ideals in a prime ring.

In this article, we have shown that every Jordan derivation of a 2-torsion free completely semiprime $\Gamma$-ring with the condition $a \alpha b \beta c=a \beta b a c, \forall a, b, c \in \mathrm{M}$ and $\alpha, \beta \in \Gamma$, is a derivation of $\mathrm{M}$.

Let $\mathrm{M}$ and $\Gamma$ be additive abelian groups. If there is a mapping $\mathrm{M} \times \Gamma \times \mathrm{M} \rightarrow \mathrm{M}$ sending $(x, a, y)$ into $x a y$ such that the conditions

(i) $(x+y) \alpha z=x \alpha z+y \alpha z, x(\alpha+\beta) y=x \alpha y+x \beta y, x \alpha(y+z)=x \alpha y+x \alpha z$ and

(ii) $\quad(x \alpha y) \beta z=x \alpha(y \beta z)$

are satisfied $\forall x, y, z \in \mathrm{M}$ and $\alpha, \beta \in \Gamma$, then $\mathrm{M}$ is called a $\Gamma$-ring. This definition is due to Barnes [1]. A $\Gamma$-ring $\mathrm{M}$ is 2-torsion free if $2 a=0(a \in \mathrm{M})$ implies $a=0$. Besides $\mathrm{M}$ is called a semiprime $\Gamma$-ring if $a \Gamma \mathrm{M} \Gamma a=0$ (with $a \in \mathrm{M}$ ) implies $a=0$. And, $\mathrm{M}$ is called completely semiprime if $a \Gamma a=0(a \in \mathrm{M})$ implies $a=0$. Note that every completely semiprime $\Gamma$-ring is clearly a semiprime $\Gamma$-ring. We define $[a, b]_{a}$ by $a a b-b a a$ which is known as a commutator of $a$ and $b$ with respect to $\alpha$. Let M be a $\Gamma$-ring. An additive mapping $d: \mathrm{M} \rightarrow \mathrm{M}$ is called a derivation if $d(a a b)=d(a) a b+\operatorname{aad}(b), \forall a, b \in \mathrm{M}$ and $\alpha \in \Gamma$. And $d: \mathrm{M} \rightarrow \mathrm{M}$ is called a Jordan derivation if $d(a \alpha a)=d(a) \alpha a+\operatorname{a\alpha d}(a)$, $\forall a \in \mathrm{M}$ and $\alpha \in \Gamma$. Throughout the article, we use the condition $a \alpha b \beta c=a \beta b \alpha c,(a, b, c$ $\in \mathrm{M}$ and $\alpha, \beta \in \Gamma)$ and refer to this condition as (*). 


\section{Some Consequences of Jordan Derivations on Completely Semiprime $\Gamma$-Ring}

In this section, we develop some useful consequences regarding the Jordan derivation of a 2 -torsion free completely semiprime $\Gamma$-ring which are very much needed for proving the main result.

Lemma 2.1 Let $\mathrm{M}$ be a $\Gamma$-ring and let $\mathrm{d}$ be a Jordan derivation of $\mathrm{M}$. Then $\forall a, b, c \in \mathrm{M}$ and $\alpha, \beta \in \Gamma$, the following statements hold:

$$
\begin{array}{ll}
\text { (i) } & d(a \alpha b+b \alpha a)=d(a) a b+d(b) \alpha a+a \alpha d(b)+b \alpha d(a) . \\
\text { (ii) } & d(a \alpha b \beta a+a \beta b \alpha a)=d(a) \alpha b \beta a+d(a) \beta b \alpha a+a \alpha d(b) \beta a+a \beta d(b) \alpha a+a \alpha b \beta d(a)+ \\
& a \beta b \alpha d(a) .
\end{array}
$$

In particular, if $\mathrm{M}$ is 2-torsion free and $\mathrm{M}$ satisfies the condition (*), then

(iii) $\quad d(a a b \beta a)=d(a) a b \beta a+a a d(b) \beta a+a a b \beta d(a)$.

(iv) $\quad d(a \alpha b \beta c+c a b \beta a)=d(a) a b \beta c+d(c) a b \beta a+a \alpha d(b) \beta c+c a d(b) \beta a+a a b \beta d(c)+$ $\operatorname{cab\beta d}(a)$.

Definition 1. Let $d$ be a Jordan derivation of a $\Gamma$-ring M. Then $\forall a, b \in \mathrm{M}$ and $\alpha \in \Gamma$, we define $G_{a}(a, b)=d(a \alpha b)-d(a) \alpha b-a a d(b)$. Thus we have $G_{a}(b, a)=d(b a a)-d(b) \alpha a-$ $\operatorname{bad}(a)$.

Lemma 2.2 Let $d$ be a Jordan derivation of a $\Gamma$-ring M. Then $\forall a, b, c \in \mathrm{M}$ and $\alpha, \beta \in \Gamma$, the following statements hold:

(i) $G_{a}(b, a)=-G_{a}(a, b)$; (ii) $G_{a}(a+b, c)=G_{a}(a, c)+G_{a}(b, c)$;

(ii) $G_{a}(a, b+c)=G_{a}(a, b)+G_{a}(a, c)$; (iv) $G_{a+\beta}(a, b)=G_{a}(a, b)+G_{\beta}(a, b)$.

Remark. $d$ is a derivation of a $\Gamma$-ring $\mathrm{M}$ if and only if $G_{a}(a, b)=0, \forall a, b \in \mathrm{M}$ and $\alpha \in \Gamma$.

Lemma 2.3 Let $M$ be a 2-torsion free $\Gamma$-ring satisfying the condition $\left(^{*}\right)$ and let $d$ be a Jordan derivation of $M$. Then

$G_{a}(a, b) \beta[a, b]_{\alpha}+[a, b]_{a} \beta G_{a}(a, b)=0, \forall a, b \in M$ and $\alpha, \beta \in \Gamma$.

Proof. (i) For any $a, b \in \mathrm{M}$ and $\alpha, \beta \in \Gamma$, we have by using Lemma 2 .1(i)

$$
\begin{aligned}
\mathrm{W} & =d(a \alpha b \beta b \alpha a+b \alpha a \beta a \alpha b) \\
& =d((a \alpha b) \beta(b a a)+(b \alpha a) \beta(a \alpha b)) \\
& =d(a \alpha b) \beta b \alpha a+a \alpha b \beta d(b \alpha a)+d(b \alpha a) \beta a a b+\operatorname{baa\alpha d}(a a b)
\end{aligned}
$$

On the other hand by using Lemma 2 .1(iii)

$$
\begin{aligned}
\mathrm{W}= & d(a \alpha(b \beta b) \alpha a+b \alpha(a \beta a) \alpha b) \\
= & d(a \alpha(b \beta b) \alpha a)+d(b a(a \beta a) \alpha b) \\
= & d(a) \alpha b \beta b \alpha a+a \alpha d(b \beta b) \alpha a+a \alpha b \beta b \alpha d(a)+d(b) \alpha a \beta a \alpha b+b \alpha d(a \beta a) \alpha b+b a a \beta a \alpha d(b) \\
= & d(a) \alpha b \beta b \alpha a+a \alpha d(b) \beta b \alpha a+a \alpha b \beta d(b) \gamma b \alpha a+a \alpha b \beta d(b) \alpha a+\operatorname{a\alpha b\beta b\alpha d}(a)+ \\
& d(b) \alpha a \beta a \alpha b+b \alpha d(a) \beta a \alpha b+b \alpha a \beta d(b) \gamma a \alpha b+b \alpha a \beta d(a) a b+b \alpha a \beta a \alpha d(b)
\end{aligned}
$$


Equating two expressions for $\mathrm{W}$ and canceling the like terms from both sides, we get

$d(a \alpha b) \beta b \alpha a+a a b \beta d(b \alpha a)+d(b \alpha a) \beta a \alpha b+b \alpha a \beta d(a \alpha b)$

$=d(a) \alpha b \beta b \alpha a+a \alpha d(b) \beta b \alpha a+a \alpha b \beta d(b) \alpha a+a a b \beta b \alpha d(a)+d(b) \alpha a \beta a \alpha b+$

$\operatorname{bad}(a) \beta a \alpha b+\operatorname{baa\beta d}(a) a b+\operatorname{baa\beta a\alpha d}(b)$

This gives $d(a \alpha b) \beta b \alpha a-d(a) \alpha b \beta b \alpha a-a \alpha d(b) \beta b \alpha a+d(b \alpha a) \beta a \alpha b-d(b) \alpha a \beta a \alpha b-$ $b \beta d(a) \beta a \alpha b+a a b \beta d(b \alpha a)-a a b \beta d(b) \alpha a-\operatorname{aab\beta bad}(a)+b a a \alpha d(a \alpha b)-b a a \beta d(a) a b-$ $\operatorname{baa} \beta a \alpha d(b)=0$

This implies that $(d(a \alpha b)-d(a) a b-a \alpha d(b)) a b a a+(d(b a a)-d(b) \alpha a-b a d(a)) \alpha a \alpha b+$ $a \alpha b \alpha(d(b \alpha a)-d(b) \alpha a-b \alpha d(a))+b \alpha a \alpha(d(a \alpha b)-d(a) a b-a \alpha d(b))=0$

Now using the Definition 1, we obtain

$G_{a}(a, b) \beta b a a+G_{a}(b, a) \beta a \alpha b+a a b \beta G_{a}(b, a)+b a a \beta G_{a}(a, b)=0$

This implies that $G_{a}(a, b) \beta[a, b]_{a}+[a, b]_{\alpha} \beta G_{a}(a, b)=0, \forall a, b \in M, \alpha, \beta \in \Gamma$.

Lemma 2.4 Let $\mathrm{M}$ be a 2-torsion free completely semiprime $\Gamma$-ring and let $a, b \in \mathrm{M}, \alpha \in \Gamma$.

If $a a b+b a a=0$, then $a a b=0=b a a$.

Proof. Let $\delta \in \Gamma$ be any element.

Using the relation $a \alpha b=-b \alpha a$ repeatedly, we get

$(a \alpha b) \delta(a \alpha b)=-(b a a) \delta(a a b)=-(b a(a \delta) a) a b=(a(\alpha a \delta) b) a b$

$$
=a \alpha(a \delta b) \alpha b=-a \alpha(b \delta a) a b=-(a a b) \delta(a a b)
$$

This implies, $2((a a b) \delta(a a b))=0$.

Since $\mathrm{M}$ is 2-torsion free, $(a \alpha b) \delta(a \alpha b)=0$

Therefore, $(a a b) \Gamma(a a b)=0$

By the completely semiprimeness of $\mathrm{M}$, we get $a a b=0$

Similarly, it can be shown that $b a a=0$.

Corollary 2.1 Let $M$ be a 2-torsion free completely semiprime $\Gamma$-ring satisfying the condition (*) and let $d$ be a Jordan derivation of M. Then $\forall a, b \in \mathrm{M}$ and $\alpha, \beta \in \Gamma$ :

(i) $G_{a}(a, b) \beta[a, b]_{a}=0 ; \quad(i i)[a, b]_{a} \beta G_{a}(a, b)=0$

Proof. Applying the result of Lemma 2.4 in that of Lemma 2.3, we obtain these results.

Lemma 2.5 Let $M$ be a 2-torsion free completely semiprime $\Gamma$-ring satisfying the condition (*) and let $d$ be a Jordan derivation of M. Then $\forall a, b, x, y \in \mathrm{M}$ and $\alpha, \beta, \gamma \in \Gamma$ :

(i) $G_{a}(a, b) \beta[x, y]_{a}=0 ;(i i)[x, y]_{a} \beta G_{a}(a, b)=0$

(iii) $G_{a}(a, b) \beta[x, y]_{y}=0$; (iv) $[x, y]_{\gamma} \beta G_{a}(a, b)=0$

Proof. (i) If we substitute $a+x$ for $a$ in the Corollary 2.1 (v), we get $G_{a}(a+x, b) \beta[a+x, b]_{a}=0$ 
Thus $G_{a}(a, b) \beta[a, b]_{a}+G_{a}(a, b) \beta[x, b]_{a}+G_{a}(x, b) \beta[a, b]_{a}+G_{a}(x, b) \beta[x, b]_{a}=0$

By using Corollary 2.1 (v), we have $G_{a}(a, b) \beta[x, b]_{a}+G_{a}(x, b) \beta[a, b]_{a}=0$

Thus, we obtain

$\left(G_{a}(a, b) \beta[x, b]_{a}\right) \beta\left(G_{a}(a, b) \beta[x, b]_{a}\right)=-G_{a}(a, b) \beta[x, b]_{a} \beta G_{a}(x, b) \beta[a, b]_{a}=0$

Hence, by the completely semiprimeness of M, we get $G_{a}(a, b) \beta[x, b]_{a}=0$

Similarly, by replacing $b+y$ for $b$ in this result, we get $G_{a}(a, b) \beta[x, y]_{a}=0$

(ii) Proceeding in the same way as described above by the similar replacements successively

in Corollary 2.1(vi), we obtain $[x, y]_{\gamma} \beta G_{a}(a, b)=0, \forall a, b, x, y \in M, \alpha, \beta \in \Gamma$

(iii) Replacing $\alpha+\gamma$ for $\alpha$ in (i), we get $G_{a+\gamma}(a, b) \beta[x, y]_{a+\gamma}=0$

This implies $\left(G_{a}(a, b)+G_{\gamma}(a, b)_{a}\right) \beta\left([x, y]_{\alpha}+[x, y]_{\gamma}\right)=0$

Therefore $G_{a}(a, b) \beta[x, y]_{a} G_{a}(a, b) \beta[x, y]_{\gamma}+G_{\gamma}(a, b) \beta[x, y]_{a}+G_{\gamma}(a, b) \beta[x, y]_{\gamma}=0$

Thus by using Corollary 2.1 (vi), we get $G_{a}(a, b) \beta[x, y]_{\gamma}+G_{\gamma}(a, b) \beta[x, y]_{a}=0$

Thus, we obtain

$\left(G_{a}(a, b) \beta[x, y]_{\gamma}\right) \beta\left(G_{a}(a, b) \beta[x, y]_{\gamma}\right)=-G_{a}(a, b) \beta[x, y]_{\gamma} \beta G_{\gamma}(a, b) \beta[x, y]_{a}=0$

Hence, by the completely semiprimeness of $\mathrm{M}$, we obtain $G_{a}(a, b) \beta[x, y]_{\gamma}=0$

(iv) As in the proof of (iii), the similar replacement in (ii) produces (iv).

Lemma 2.6 Every completely semiprime $\Gamma$-ring contains no nonzero nilpotent ideal.

Corollary 2.2 Completely Semiprime $\Gamma$-ring has no nonzero nilpotent element.

Lemma 2.7 The center of a completely semiprime $\Gamma$-ring does not contain any nonzero nilpotent element.

\section{Jordan Derivations on Completely Semiprime $\Gamma$-ring}

We are now ready to prove our main result as follows:

Theorem 3.1 Let $M$ be a 2-torsion free completely semiprime $\Gamma$-ring satisfying the condition $(*)$ and let $d$ be a Jordan derivation of M. Then $d$ is a derivation of M.

Proof. Let $d$ be a Jordan derivation of a 2-torsion free completely semiprime $\Gamma$-ring $\mathrm{M}$ and let $a, b, y \in \mathrm{M}$ and $\alpha, \beta, \gamma \in \Gamma$. Then by Lemma 2.5 (iii), we get

$$
\left[G_{a}(a, b), y\right]_{\beta} \gamma\left[G_{a}(a, b), y\right]_{\beta}=\left(G_{a}(a, b) \beta y-y \beta G_{a}(a, b)\right) \gamma\left[G_{a}(a, b), y\right]_{\beta}
$$

$=G_{a}(a, b) \beta y \gamma\left[G_{a}(a, b), y\right]_{\beta}-y \beta G_{a}(a, b) \gamma\left[G_{a}(a, b), y\right]_{\beta}=0$

Since $\beta y \gamma \in \mathrm{M}$ and $G_{a}(a, b) \in M, \forall a, b, y \in \mathrm{M}$ and $\alpha, \beta, \gamma \in \Gamma$.

By the completely semiprimeness of $\mathrm{M},\left[G_{a}(a, b), y\right]_{\beta}=0$, where $G_{a}(a, b) \in M, \forall a, b$, $y \in M$ and $\alpha, \beta \in \Gamma$. 
Therefore, $G_{a}(a, b) \in \mathrm{Z}(\mathrm{M})$, the center of $\mathrm{M}$.

Now, by Lemma 2.5(iii), we have $G_{a}(a, b) \gamma[x, y]_{\beta}=0$

Also, by Lemma 2.5(iv), we have $[x, y]_{\beta} \gamma G_{a}(a, b)=0$

Thus, we have

$$
\begin{aligned}
2 G_{a}(a, b) \gamma G_{a}(a, b) & =G_{a}(a, b) \gamma\left(G_{a}\left(a, b+G_{a}(a, b)\right)\right. \\
& =G_{a}(a, b) \gamma\left(G_{a}(a, b)-G_{a}(b, a)\right) \\
& =G_{a}(a, b) \gamma(d(a \alpha b)-d(a) \alpha b-\operatorname{a\alpha d}(b)-d(b \alpha a)+d(b) \alpha a+b \alpha d(a)) \\
& =G_{a}(a, b) \gamma(d(a \alpha b-b \alpha a)+(b \alpha d(a)-d(a) \alpha b)+(d(b) \alpha a-\operatorname{a\alpha d}(b))) \\
& =G_{a}(a, b) \gamma\left(d\left([a, b]_{a}\right)+[b, d(a)]_{a}+[d(b), a]_{a}\right) \\
& =G_{a}(a, b) \gamma d\left([a, b]_{a}\right)-G_{a}(a, b) \gamma[d(a), b]_{a}-G_{a}(a, b) \gamma[a, d(b)]_{a}
\end{aligned}
$$

Since $d(a), d(b) \in \mathrm{M}$, by using Lemma 2.5(i) and (1), we get

$$
G_{a}(a, b) \gamma[d(a), b]_{a}=G_{a}(a, b) \gamma[a, d(b)]_{a}=0
$$

Thus $2 G_{a}(a, b) \gamma G_{a}(a, b)=G_{a}(a, b) \gamma d\left([a, b]_{a}\right)$

Adding (1) and (2), we obtain $G_{a}(a, b) \gamma[x, y]_{\beta}+[x, y]_{\beta} \gamma G_{a}(a, b)=0$.

Then by Lemma 2 .1(i) with the use of (1), we have

$$
\begin{aligned}
0 & =d\left(G_{a}(a, b) \gamma[x, y]_{\beta}+[x, y]_{\beta} \gamma G_{a}(a, b)\right) \\
& =d\left(G_{a}(a, b)\right) \gamma[x, y]_{\beta}+d\left([x, y]_{\beta}\right) \gamma G_{a}(a, b)+G_{a}(a, b) \gamma d\left([x, y]_{\beta}\right)+[x, y]_{\beta} \gamma d\left(G_{a}(a, b)\right) \\
& =d\left(G_{a}(a, b)\right) \gamma[x, y]_{\beta}+2 G_{a}(a, b) \gamma d\left([x, y]_{\beta}\right)+[x, y]_{\beta} \gamma d\left(G_{a}(a, b)\right)
\end{aligned}
$$

Since $G_{a}(a, b) \in \mathrm{Z}(\mathrm{M})$ and therefore $d\left([x, y]_{\beta}\right) G_{a}(a, b)=G_{a}(a, b) d\left([x, y]_{\beta}\right)$

Hence, we get

$2 G_{a}(a, b) \gamma d\left([x, y]_{\beta}\right)=-d\left(G_{a}(a, b)\right) \gamma[x, y]_{\beta}-[x, y]_{\beta} \gamma d\left(G_{a}(a, b)\right)$

Then from (3) and (4) we have

$4 G_{a}(a, b) \gamma G_{a}(a, b)=2 G_{a}(a, b) \gamma d\left([a, b]_{a}\right)=-d\left(G_{a}(a, b)\right) \gamma[a, b]_{a}-[a, b]_{a} \gamma d\left(G_{a}(a, b)\right)$

Thus we obtain

$4 G_{a}(a, b) \gamma G_{a}(a, b) \gamma G_{a}(a, b)=-d\left(G_{a}(a, b)\right) \gamma[a, b]_{a} \gamma G_{a}(a, b)$

$$
-[a, b]_{a} \gamma d\left(G_{a}(a, b)\right) \gamma G_{a}(a, b)
$$

Here, we have by Corollary 2.1 (vi) $d\left(G_{a}(a, b)\right) \gamma[a, b]_{a} \gamma G_{a}(a, b)=0$

and also, by Lemma 2.5(iv) $[a, b]_{a} \gamma d\left(G_{a}(a, b)\right) \gamma G_{a}(a, b)=0$.

Since $d\left(G_{a}(a, b)\right) \in \mathrm{M}, \forall a, b \in \mathrm{M}$ and $a \in \Gamma$.

So, we get $4 G_{a}(a, b) \gamma G_{a}(a, b) \gamma G_{a}(a, b)=0$.

Therefore, $4\left(G_{a}(a, b) \gamma\right)^{2} G_{a}(a, b)=0$. 
Since M is 2-torsion free, so we have $\left(G_{a}(a, b) \gamma\right)^{2} G_{a}(a, b)=0$

But, it follows that $G_{a}(a, b)$ is a nilpotent element of the $\Gamma$-ring $\mathrm{M}$.

Since by Lemma 2.7 , the center of a completely semiprime $\Gamma$-ring does not contain any nonzero nilpotent element, so we get $G_{a}(a, b)=0, \forall a, b \in \mathrm{M}$ and $a \in \Gamma$.

It means that, $d$ is a derivation of $\mathrm{M}$. Which is the required result.

\section{REFERENCES}

[1] Barnes, W. E., On the $\Gamma$-rings of Nobusawa, Pacific J. Math., 18(1966), 411-422.

[2] Bresar, M., Jordan derivations on semiprime rings. Proc. Amer. Math. Soc. 104(4), (1988), 1003-1004.

[3] Chakraborty, S. and Paul, A. C., On Jordan k-derivations of 2-torsionfree Prime $\Gamma$ N-rings, Punjab Univ. J. Math. 40(2008), 97-101.

[4] Haetinger, C., Higher derivations on Lie ideals, Tenclencias em Matematica Aplicada e. computacional, 3(1), (2002), 141-145.

[5] Herstein, I. N., Jordan derivations of prime rings, Proc.Amer.Math. Soc., 8(1957), 1104-110.

[6] Herstein, I. N., Topics in Ring Theory, The University of Chicago Press, Chicago, 1969.

[7] Nobusawa, N., On the generalizeation of the ring theory, Osaka J. Math., 1(1964), 81-89.

[8] Sapanci, M. and Nakajima, A., Jordan Derivations on Completely Prime $\Gamma$-Rings, Math. Japanica, 46 (1997), 47-51. 